\title{
Electron screening and excitonic condensation in double-layer graphene systems
}

\author{
Maxim Yu. Kharitonov ${ }^{1}$ and Konstantin B. Efetov ${ }^{1,2}$ \\ 1 Theoretische Physik III, Ruhr-Universität Bochum, Germany \\ ${ }^{2}$ L.D. Landau Institute for Theoretical Physics, Moscow, Russia
}

(Dated: October 26, 2018)

\begin{abstract}
We theoretically investigate the possibility of excitonic condensation in a system of two graphene monolayers separated by an insulator, in which electrons and holes in the layers are induced by external gates. In contrast to the recent studies of this system, we take into account the screening of the interlayer Coulomb interaction by the carriers in the layers, and this drastically changes the result. Due to a large number of electron species in the system (two projections of spin, two valleys, and two layers) and to the suppression of backscattering in graphene, the maximum possible strength of the screened Coulomb interaction appears to be quite small making the weak-coupling treatment applicable. We calculate the mean-field transition temperature for a clean system and demonstrate that its highest possible value $T_{c}^{\max } \sim 10^{-7} \epsilon_{F} \lesssim 1 \mathrm{mK}$ is extremely small ( $\epsilon_{F}$ is the Fermi energy). In addition, any sufficiently short-range disorder with the scattering time $\tau \lesssim \hbar / T_{c}^{\max }$ would suppress the condensate completely. Our findings renders experimental observation of excitonic condensation in the above setup improbable even at very low temperatures.
\end{abstract}

PACS numbers: 73.63.-b, 72.15.Rn, 81.05.Uw

\section{INTRODUCTION AND MAIN RESULT}

The possibility of excitonic condensation (EC) in metallic systems was originally proposed by Keldysh and Kopaev [1] for semimetals with overlapping conduction and valence bands. They have shown that the attractive Coulomb interaction between electrons and holes leads to an instability towards formation of bound electron-hole pairs analogous to the Cooper instability in superconductors. Somewhat later, it was suggested [2] that EC could be realized in a double-layer system of spatially separated electrons and holes. Experimental efforts towards the observation of EC were mainly concentrated on semiconductor double quantum well systems [3, 4, 5, 6] and experimental data speak in favor of the existence of EC in electron-hole bilayers [3, 4, 5] and electron-electron bilayers in the quantum Hall regime [6].

Since the carrier density in graphene, including its polarity, can effectively be controlled by various means, graphene-based systems may also seem attractive for the realization of EC. Indeed, several ideas on how one could obtain EC in graphene have been suggested recently. One possible way to create interacting electrons and holes is to apply a strong in-plane magnetic field to a single layer of graphene 7]. Such a magnetic field acts on the spins of the carriers only, and the Zeeman splitting creates electrons with one spin polarization and holes with the opposite polarization in an initially neutral sample. A detailed theory of EC in such a setup has been developed in Ref. 7].

A double-layer graphene system (Fig. 1) as a candidate for the observation of EC was proposed recently in Refs. [8, 9, 10]. If two graphene layers are separated by an insulator, electrons in one layer and holes in the other can be obtained by applying external gate voltage. Relatively high values of the Fermi energy $\epsilon_{F} \sim 0.3 \mathrm{eV}$ that

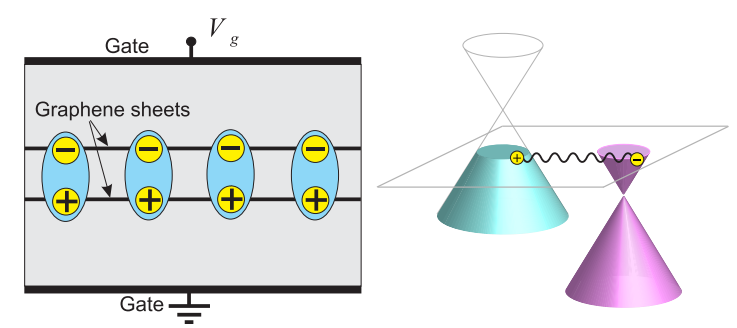

FIG. 1: Excitonic condensate in a system of two spatially separated graphene layers. Electrons and holes in the layers are induced by applying the external gate voltage.

can be achieved in graphene by using gates [11] are an obvious advantage, since $\epsilon_{F}$ serves as a high-energy scale of the effect in such a setup. Solving the gap equations numerically, the authors of Refs. [9, 10] provided an estimate $T_{B K T} \sim 0.1 \epsilon_{F}$ for the critical temperature of the Berezinski-Kosterlitz-Thouless (BKT) transition and argued that $T_{B K T}$ could thus reach room temperatures.

However, in the analysis of Refs. 9, 10], the screening of the Coulomb interactions by the carriers in the graphene layers was not taken into account. Clearly, the two-dimensional screening cloud formed around a probe charge in a graphene sheet screens the field of the charge both in the off-plane and in-plane directions, although not identically. Therefore, screening affects not only the intralayer but also the interlayer Coulomb interaction in the double-layer setup.

In this paper, we demonstrate that taking screening into account is essential as it drastically reduces the transition temperature compared to the estimate obtained in Refs [9, 10] neglecting screening. In fact, screening sets the upper bound for the interaction strength, yielding for the maximum possible value of the dimensionless 
coupling constant

$$
\lambda^{\max }=\frac{1}{N}=\frac{1}{8} .
$$

Here $N=N_{s} N_{v} N_{l}=2^{3}=8$ is the total number of electron species in the system originating from two projections of $\operatorname{spin}\left(N_{s}=2\right)$, two valleys $\left(N_{v}=2\right)$, and two layers $\left(N_{l}=2\right)$. Moreover, the chiral nature of quasiparticles in graphene leads to the suppression of backscattering. Consequently, the maximum interaction strength $\lambda_{c}^{\max }$ that determines the transition temperature appears to be actually two times smaller than $\lambda^{\max }$,

$$
\lambda_{c}^{\max }=\frac{\lambda^{\max }}{2}=\frac{1}{16} .
$$

As follows from Eqs. (1) and (21), the large number of electron species and suppression of backscattering make the maximum possible value $\lambda_{c}^{\max }$ of the interaction strength numerically quite small. This justifies the applicability of the weak-coupling BCS approach to the problem, since $1 / 16$ can safely be considered as a small parameter. As a result, for the highest possible value of the mean-field transition temperature we obtain

$$
T_{c}^{\max } \approx \exp \left(-1 / \lambda_{c}^{\max }\right) \epsilon_{F}=\exp (-16) \epsilon_{F} \approx 10^{-7} \epsilon_{F} .
$$

This is the highest possible value of the critical temperature of the excitonic condensation that could be achieved in a perfectly clean double-layer graphene system. Entering Eq. (3) as the exponent, the large value of the inverse interaction strength $1 / \lambda_{c}^{\max }=16$ results in a drastic reduction of the transition temperature. In order to achieve the maximum value (3), the interlayer distance $d$ must be much smaller than the Debye screening length $\varkappa^{-1}$,

$$
2 \varkappa d \ll 1
$$

The most optimistic estimate would thus be $T_{c}^{\max } \sim$ $1 \mathrm{mK}$ for $\epsilon_{F} \sim 0.3 \mathrm{eV}$ and would require $d \lesssim 0.2 \mathrm{~nm}$.

\section{CALCULATIONS}

We now present the details of derivation of Eq. (3). The bare strength of Coulomb interactions in graphene is not that small. For $\mathrm{SiO}_{2}$ used as an insulator embedding graphene sheets, typical values of the dimensionless coupling constant are $r_{s}=e^{2} /(\varepsilon v) \sim 1$ ( $\varepsilon$ is the dielectric constant of the insulator and $v$ is the velocity of the Dirac spectrum, we set $\hbar=1$ throughout this section). This questions the applicability of the weak-coupling approach to the problem of EC, suggesting, at the same time, that the transition temperature could be quite high [9, 10].

It is known, however, that in a fermionic system with a large number $N \gg 1$ of independent fermionic species the interactions are significantly weakened. Physically, large
$N$ makes screening very effective, since all $N$ species participate in the screening of interactions between fermions of each particular species. Screening reduces the coupling constant from $r_{s}$ to the value $1 / N \ll 1: r_{s} \rightarrow 1 / N$. Effectively, the system becomes weakly interacting, despite of the fact that the bare Coulomb interactions may be not weak $\left(r_{s} \gtrsim 1\right)$.

The large- $N$ approximation was already used for a single-layer graphene [7, 12, 13] before. In a single layer, the number of species is equal to $N_{1}=N_{s} N_{v}=4$ due to two projections of spin $\left(N_{s}=2\right)$ and two valleys $\left(N_{v}=2\right)$. This value is not exceptionally large, but does give hope that the large- $N$ approach adequately describes graphene physics. In a double-layer system the situation is better: since each electron can belong to either one of the layers, one has an additional "which-layer" degree of freedom $\left(N_{l}=2\right)$ making the total number of species $N=N_{s} N_{v} N_{l}=8$. It would already be quite reasonable to treat $N=8$ as a large parameter. Therefore, large$N$ approximation seems to be particularly suitable for a double-layer graphene system and is expected to provide good quantitative predictions.

Below we employ the large- $N$ approach to the doublelayer graphene system (Fig. 11) treating $N=8$ as a large parameter and calculate the mean-field critical temperature $T_{c}$ of EC. The calculations follow closely those of Ref. [7]. Of course, the mean-field treatment of twodimensional systems is not necessarily a good one due to strong thermal fluctuations. It is, however, sufficient for our purposes, as our main goal is to demonstrate that already the mean-field critical temperature is extremely low. The temperature $T_{B K T}$ of the actual BKT transition can only be lower than the mean-field $T_{c}$ we calculate here.

Within the large- $N$ approximation, the diagrammatic series for the effective interaction between the electrons is identical to that of the random-phase approximation (RPA), which describes linear screening. For the problem at hand, the relevant transfer momenta $q$ are in the range $0 \leq q \leq 2 p_{F}$, with $p_{F}=\epsilon_{F} / v$ the Fermi momentum, and one has to use the exact expression for the polarization operator (and not its limit form for $q \ll p_{F}$ ). However, the static polarization operator in graphene 14] does not depend on momentum at all in this range and equals $\Pi(\omega=0, q)=N_{s} N_{v} \nu$, where $\nu=\epsilon_{F} /\left(2 \pi v^{2}\right)$ is the density of states per one valley and one spin. As a result, for the screened interlayer Coulomb interaction in the momentum space we obtain

$$
V(q)=\frac{2 \pi e_{*}^{2} \exp (-q d)}{q+2 \varkappa+\varkappa^{2}[1-\exp (-q d)] / q}, q \leq 2 p_{F} .
$$

In Eq. (5), $q$ is the absolute value of the in-plane twodimensional wave vector, $d$ is the distance between the layers, $e_{*}$ is the effective electron charge screened by the insulator embedding graphene sheets, $e_{*}^{2}=e^{2} / \varepsilon$, and $\varkappa=2 \pi N_{s} N_{v} e_{*}^{2} \nu$ is the inverse Debye screening length 


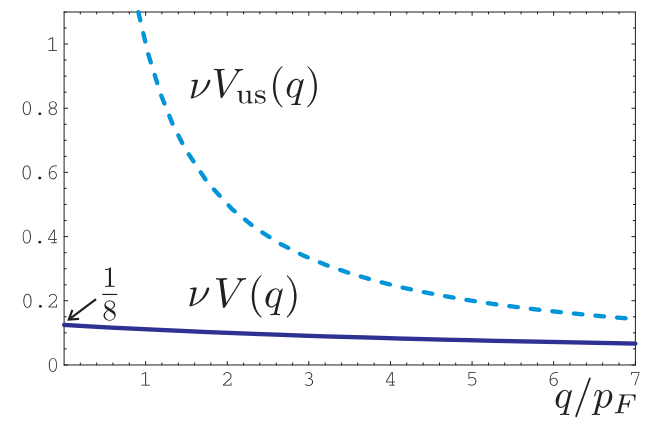

FIG. 2: The screened $V(q)$ [Eq. (5), solid line] and unscreened $V_{\mathrm{us}}(q)$ [Eq. (8), dashed line] interlayer Coulomb interaction, responsible for the excitonic instability. The values $d=0$ and $r_{s}=e^{2} /(\varepsilon v)=1$ were used, $\nu$ is the density of states. At relevant momenta $q \sim p_{F}$, the unscreened interaction potential overestimates the actual screened one by about 10 times. The screened Coulomb interaction reaches its maximum at $q=0$, the universal value (1) of which is achieved for $2 \varkappa d \ll 1$ [Eq. (4)].

in each layer. We assume the same Fermi momenta $p_{F}$ of electrons and holes (this can be achieved by tuning the gate voltage), since any difference between them would be suppressing the condensate in a way Zeeman splitting suppresses s-wave superconductivity.

The screened Coulomb interaction (5) is a decreasing function of $q$ (Fig. 2) and reaches its maximum at $q=0$,

$$
V(q=0)=\frac{2 \pi e_{*}^{2}}{2 \varkappa+\varkappa^{2} d} .
$$

The maximum of Eq. (6) is achieved, if the distance $d$ between the layers is smaller than the Debye radius [Eq. (4)], and equals

$$
V^{\max }(q=0)=\frac{1}{2 N_{s} N_{v} \nu} .
$$

The factor 2 that enters the denominator in Eq. (7) is due to "which layer" degree of freedom $\left(N_{l}=2\right)$, since each carrier can belong to either one of the layers. Equation (7) leads to Eq. (11) for the maximum value of the dimensionless coupling constant $\lambda^{\max }=\nu V^{\max }(q=0)$.

In contrast to the above calculation, in the analysis of Refs. [9, 10] the unscreened form

$$
V_{\mathrm{us}}(q)=2 \pi e_{*}^{2} \exp (-q d) / q
$$

of the Coulomb interaction $V(q)$ [Eq. (5)] was used, see Eq. (4) in Ref. 9] and inline formulas before Eq. (2) in Ref. [10]. As seen from Eqs. (5) and (8) and Fig. 2, the unscreened form $V_{\mathrm{us}}(q)$ is valid for $q \gg 2 \varkappa=N r_{s} p_{F}$, but significantly overestimates the actual screened interaction $V(q)$ for relevant momenta $q \leq 2 p_{F}$. For the value $r_{s}=1$ used in Ref. 9] and typical for $\mathrm{SiO}_{2}$ as an insulator, one obtains $V_{\mathrm{us}}\left(p_{F}\right) \approx 9 V\left(p_{F}\right)$. Using the unscreened form of the Coulomb interaction in Refs. 9, 10] resulted in the estimate $T_{B K T} \sim 0.1 \epsilon_{F}$ and, as it appears, led an overestimation of $T_{B K T}$ by a factor $10^{6}$, see Eq. (3).

In order to obtain the mean-field transition temperature $T_{c}$, we derive the linearized gap equation for the order parameter

$$
\hat{\Delta}\left(\mathbf{r}-\mathbf{r}^{\prime}\right)=V\left(\mathbf{r}-\mathbf{r}^{\prime}\right)\left\langle\hat{\phi}_{e}(\mathbf{r}) \hat{\phi}_{h}^{\dagger}\left(\mathbf{r}^{\prime}\right)\right\rangle .
$$

Here, $V\left(\mathbf{r}-\mathbf{r}^{\prime}\right)$ is the interaction (5) in the coordinate space and $\hat{\phi}_{e, h}$ are the Dirac spinor fields of electrons and holes in the graphene sheets. The matrix structure of the order parameter in the sublattice space is predetermined by chirality, but can be arbitrary in the valley and spin spaces. Using the standard BCS approach, we arrive at the linearized gap equation

$$
\hat{\Delta}(\mathbf{n})=\nu \ln \frac{\epsilon_{F}}{T} \int \frac{\mathrm{d} \mathbf{n}^{\prime}}{2 \pi} V\left(p_{F}\left|\mathbf{n}-\mathbf{n}^{\prime}\right|\right) \hat{\mathcal{P}}\left(\mathbf{n}^{\prime}\right) \hat{\Delta}\left(\mathbf{n}^{\prime}\right) \hat{\mathcal{P}}\left(-\mathbf{n}^{\prime}\right),
$$

where the two-dimensional unit vectors $\mathbf{n}$ and $\mathbf{n}^{\prime}$ represent the direction of the electron momentum and $\hat{\mathcal{P}}(\mathbf{n})=$ $(1+\boldsymbol{\tau} \mathbf{n}) / 2$, with $\tau_{x}$ and $\tau_{y}$ the Pauli matrices in the sublattice space. The value of temperature $T$, at which a nonzero solution $\hat{\Delta}(\mathbf{n})$ to Eq. (10) appears, determines $T_{c}$. Solving Eq. (10), we obtain

$$
T_{c} \approx \exp \left(-1 / \lambda_{c}\right) \epsilon_{F},
$$

where

$$
\lambda_{c}=\nu \int_{-\pi}^{\pi} \frac{\mathrm{d} \theta}{2 \pi} V\left(2 p_{F} \sin \frac{\theta}{2}\right) \frac{1+\cos \theta}{2} .
$$

The exact numerical value $\sim 1$ of the prefactor in Eq. (11) cannot be obtained within the logarithmic accuracy of the mean-field approach. The form of Eqs. (11) and (12) and the matrix structure of the solution $\hat{\Delta}(\mathbf{n})$ in the sublattice space are identical to those in Ref. 7] [see Eqs. (5.23)-(5.31) therein]. At the same time, the form (5) of the interaction $V(q)$ is different here.

The maximum possible value $\lambda_{c}^{\max }$ [Eq. (2)] of the interaction constant $\lambda_{c}$ [Eq. (12)] and, thus, the highest possible transition temperature $T_{c}^{\max }$, [Eq. (3)], are obtained by inserting Eq. (7) into Eq. (12). This corresponds to the limit $p_{F} \ll 2 \varkappa \ll 1 / d$ [Eq. (44)], where the condition $p_{F} \ll 2 \varkappa$ is automatically satisfied, since $r_{s} \sim 1$ and $2 \varkappa / p_{F}=r_{s} N \gg 1$. The factor $(1+\cos \theta) / 2$ entering Eq. (12) is a consequence of chirality. It suppresses backscattering and reduces $\lambda_{c}^{\max }$ by a factor 2 compared to $\lambda^{\max }$ [Eq. (11)], see Eq. (2).

The obtained small value of $\lambda_{c}^{\max }=1 / 16$ justifies the very applicability of the weak-coupling BCS approach to determining $T_{c}$, within which the logarithm $\ln \left(\epsilon_{F} / T\right) \approx 16$ has to be large. Therefore, the critical temperature $T_{c}$ does exponentially depend on the inverse coupling constant $1 / \lambda_{c}$, which leads to its extremely small value [Eq. (3)] . 


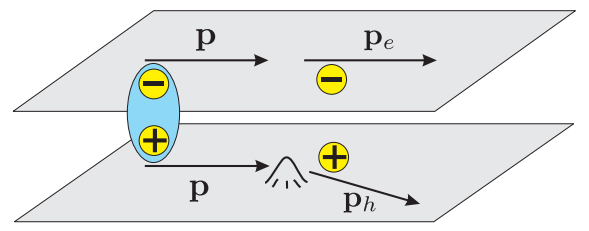

FIG. 3: Sensitivity of the excitonic condensate to the impurity scattering. Impurities with the size of potential smaller than the interlayer distance scatter electrons and holes not identically, thereby breaking electron-hole pairs and suppressing the condensate.

\section{DISCUSSION AND CONCLUSION}

Let us now discuss the obtained results. Remarkably enough, as Eqs. (1)-(3) demonstrate, the specifics of the graphene spectrum (chirality and valley degrees of freedom) appears to be very unfavorable for the realization of EC in graphene-based devices. At the same time, this is not so for double-layer systems based on materials with "conventional" metallic spectrum, such as, e.g., $\mathrm{GaAs} / \mathrm{Al}_{x} \mathrm{Ga}_{1-x} \mathrm{As}$ heterostructures used so far experimentally $[3,44,5,6]$. Indeed, in such systems the maximum interaction strength $\lambda_{c}^{\max }=1 /\left(N_{s} N_{l}\right)=1 / 4$ is four times larger than in graphene [Eq. (2)] due to the absence of the valley space and chirality. This value is not that small and the system could be on the verge of the weak-coupling limit. Therefore, one does not get such a small value of the transition temperature as we obtained for graphene.

It is also instructive to mention for comparison that in a single-layer graphene subject to the in-plane magnetic field [7] one obtains $\lambda_{c}^{\max }=1 /\left(2 N_{s} N_{v}\right)=1 / 8$ for the interaction constant, since the system consists of only one layer $\left(N_{l}=1\right)$ [see Eq. (5.29b) in Ref. [7]], and the exponential factor $\exp \left(-\lambda_{c}^{\max }\right)=\exp (-8) \approx 3 \cdot 10^{-4}$ in Eq. (3) is not as small. However, the Zeeman splitting energy enters Eq. (3) instead of $\epsilon_{F}$, which cannot be extremely high even for experimentally very high magnetic fields $B$. For $B \approx 40 \mathrm{~T}$ one can estimate $T_{c}^{\max } \sim 20 \mathrm{mK}$.

There is another factor that is unfavorable for the realization of EC in double-layer systems. Namely, the excitonic condensate is sensitive to the impurity scattering $[2,15]$. Since the bound electron and hole carry the same momentum $\mathbf{p}$, any scattering process that changes the momentum of electron and hole not identically, i.e., $\mathbf{p} \rightarrow \mathbf{p}_{e}$ for electron and $\mathbf{p} \rightarrow \mathbf{p}_{h}$ for hole, so that $\mathbf{p}_{e} \neq \mathbf{p}_{h}$, breaks the electron-hole pair (see Fig. 3). This is the case for any impurities with the range of the scattering potential less than the interlayer distance $d$, since the potential of such impurities differs in the two layers. The effect of the impurity scattering on the excitonic condensate was studied analytically for conventional systems in Refs. [2, 15] and the theory is analogous to AbrikosovGorkov's theory for magnetic impurities in superconduc- tors. This approach has been very recently applied to graphene in Ref. [16]. The main result of this study is that sufficiently short-range impurities with the scattering time $\tau$ destroy the excitonic condensate completely as soon as

$$
\hbar / \tau \gtrsim T_{c}
$$

where $T_{c}$ is the transition temperature of the ideally clean system. Equivalently, for the condensate to exist, electron momentum has to be conserved at the scale of the correlation length $\hbar v / T_{c}$. Since the mean free path $v \tau \sim 1 \mu \mathrm{m}$ of the order of the typical size of graphene samples corresponds to $\hbar / \tau \sim 1 \mathrm{~K}$, considering the values $T_{c}^{\max } \sim 1 \mathrm{mK}$ obtained, the condensate should be completely suppressed at any temperature $T \leq T_{c}^{\max }$ even in the ballistic samples due to the boundary scattering.

In conclusion, we have studied the possibility of the excitonic condensation in double-layer graphene systems. We have demonstrated that in order to properly determine the transition temperature, it is essential to take the screening of the coupling interlayer Coulomb interaction into account. The specifics of the graphene spectrum (chirality and valley degrees of freedom) leads to a smaller interaction strength than in conventional semiconductors and to an extremely small value $\lesssim 1 \mathrm{mK}$ of the transition temperature. This makes graphene-based systems disadvantageous for the observation of the excitonic condensation.

After the present work was completed, we became aware of Ref. [17], in which Eqs. (11) and (12) were obtained. However, contrary to our main argument, that these equations are valid and provide good quantitative prediction also for moderate to strong Coulomb interactions $\left(r_{s} \gtrsim 1\right)$, it was stated in Ref. [17] that they should apply in the weak coupling limit $\left(r_{s} \ll 1\right.$ or $\left.p_{F} d \gg 1\right)$ only, whereas for $r_{s} \sim 1$, analogously to Refs. [9, 10], one could expect high $T_{c}$ of the order of room temperatures.

We thank Anatoly F. Volkov and Sergey V. Syzranov for useful discussions. Financial support of SFB Transregio 12 is greatly appreciated.

[1] L. V. Keldysh and Yu. V. Kopaev, Sov. Phys. Solid State 6, 2219 (1965).

[2] Yu. E. Lozovik and V. I. Yudson, JETP Lett. 22, 274 (1975); Sov. Phys. JETP 44, 389 (1975); Solid State Commun. 19, 391 (1976); Solid State Commun. 21, 211 (1977).

[3] U. Sivan, P. M. Solomon, and H. Shtrikman, Phys. Rev. Lett. 68, 1196 (1992).

[4] L. V. Butov, A. Zrenner, G. Abstreiter, G. Böhm, and G. Weimann, Phys. Rev. Lett. 73, 304 (1994).

[5] M. Bayer, V. B. Timofeev, F. Faller, T. Gutbrod, and A. Forchel, Phys. Rev. B 54, 8799 (1996).

[6] I. B. Spielman, J. P. Eisenstein, L. N. Pfeiffer, and K. W. West, Phys. Rev. Lett. 84, 5808 (2000); Phys. Rev. Lett. 
87, 036803 (2001); J. P. Eisenstein and A. H. MacDonald, Nature 432, 691 (2004).

[7] I. L. Aleiner, D. E. Kharzeev, and A. M. Tsvelik, Phys. Rev. B 76, 195415 (2007).

[8] J.-J. Su and A.H. MacDonald, arXiv:0801.3694, unpublished.

[9] H. Min, R. Bistritzer, J.-J. Su, and A.H. MacDonald, arXiv:0802.3462, unpublished.

[10] C.-H. Zhang and Y. N. Joglekar, Phys. Rev. B 77, 233405 (2008).

[11] K.S. Novoselov et al., Nature 438, 197 (2005).
[12] D. T. Son, Phys. Rev. B 75, 235423 (2007).

[13] M. S. Foster and I. L. Aleiner, Phys. Rev. B 77, 195413 (2008).

[14] B. Wunsch, T. Stauber, F. Sols, and F. Guinea, New J. Phys. 8, 318 (2006).

[15] J. Zittartz, Phys. Rev. 164, 575 (1967).

[16] R. Bistritzer and A.H. MacDonald, arXiv:0808.1310, unpublished.

[17] Yu. E. Lozovik, S. P. Merkulova, and A. A. Sokolik, Uspekhi Fiz. Nauk 178, 757 (2008), in Russian. 